\title{
Evolution from increased cardiac mechanical function towards cardiomyopathy in the obese rat due to unbalanced high fat and abundant equilibrated diets
}

\author{
Evangelia Mourmoura ${ }^{1,2}$, Valérie Chaté ${ }^{1,2}$, Karine Couturier ${ }^{1,2}$, Corinne Malpuech-Brugère ${ }^{3}$, \\ Kasra Azarnoush $^{3,4}$ and Luc Demaison ${ }^{1,2,3, \star}$ \\ 1 Laboratoire de Bioénergétique Fondamentale et Appliquée, INSERM U1055, Grenoble Cedex 09, 38041, France \\ 2 Université Joseph Fourier, Laboratoire de Bioénergétique Fondamentale et Appliquée, Grenoble Cedex 09, 38041, France \\ 3 Unité de Nutrition Humaine, INRA, UMR 1019, Clermont Université, Université d'Auvergne, BP 10448, 63000 Clermont-Ferrand, \\ France \\ ${ }^{4}$ Clermont-Ferrand University Hospital, Heart Surgery Department, G. Montpied Hospital, 63000 Clermont-Ferrand, France
}

Received 7 January 2015 - Accepted 9 February 2015

\begin{abstract}
The aim of our study was to know whether high dietary energy intake (HDEI) with equilibrated and unbalanced diets in term of lipid composition modify the fatty acid profile of cardiac phospholipids and function of various cardiac cells and to know if the changes in membrane lipid composition can explain the modifications of cellular activity. Wistar rats were fed either a control or high-fat (HF) diet for 12 weeks and Zucker diabetic fatty (ZDF) rats as well as their lean littermate (ZL) a control diet between week 7 to 11 of their life. Energy intake and abdominal obesity was increased in HF-fed and ZDF rats. Circulating lipids were also augmented in both strains although hyperglycemia was noticed only in ZDF rats. HDEI induced a decrease in linoleate and increase in arachidonate in membrane phospholipids which was more pronounced in the ZDF rats compared to the HF-fed rats. In vivo cardiac function (CF) was improved in HF-fed rats whereas ex vivo cardiac function was unchanged, suggesting that environmental factors such as catecholamines stimulated the in vivo $\mathrm{CF}$. The unchanged ex vivo $\mathrm{CF}$ was associated with an increased cardiac mass which indicated development of fibrosis and/or hypertrophy. The increased in vivo $\mathrm{CF}$ was sustained by an augmented coronary reserve which was related to the cyclooxygenase pathway and accumulation of arachidonate in membrane phospholipids. In conclusion, before triggering a diabetic cardiomyopathy, HDEI stimulated the CF. The development of cardiomyopathy seems to result from fibrosis and/or hypertrophy which augments myocardial stiffness and decreases contractility.
\end{abstract}

Keywords: High dietary energy intake / abdominal adiposity / myocardial function / phospholipid composition / arachidonic acid / coronary reserve

Résumé - Évolution du stade de l'activation mécanique jusqu'à la cardiomyopathie chez le rat rendu obèse par des régimes équilibré en graisses ou riche en acides gras saturés et mono-insaturés. Notre objectif a été de savoir si l'excès calorique (EC) modifie la composition en acides gras des phospholipides et la fonction cardiaques et si ces modifications des membranes expliquent les changements d'activité des cellules cardiaques. Des rats Wistar ont été nourris soit avec un régime contrôle, soit avec un régime riche en graisse (RRG) pendant 12 semaines et des rats Zucker diabétiques gras (ZDF) ainsi que leurs homologues maigres (ZL) avec un régime contrôle entre leur $7^{\mathrm{e}}$ et $11^{\mathrm{e}}$ semaine de vie. L'ingestion calorique et l'obésité abdominale ont été augmentées chez les rats RRG et ZDF. Une hyperlipidémie a été observée dans les 2 souches, mais l'hyperglycémie n'a été observée que chez les animaux ZDF. L'EC a provoqué une diminution en linoléate et une augmentation en arachidonate dans les membranes cardiaques qui a été plus prononcée chez les rats ZDF. La fonction cardiaque (FC) in vivo a été plus élevée chez les rats RRG malgré une FC ex vivo inchangée. Les catécholamines circulantes pourraient expliquer les discordances. La stabilité de la FC ex vivo a été associée à une augmentation de la masse cardiaque suggérant le développement d'une fibrose et/ou d'une hypertrophie. L'augmentation de la FC in vivo a été soutenue par un

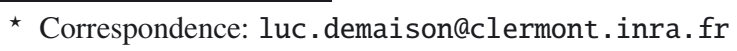


accroissement de la réserve coronaire reliée à l'activité de la cyclo-oxygénase et à l'accumulation d'arachidonate. En conclusion, avant de provoquer une cardiomyopathie, l'EC stimule la FC. La cardiomyopathie semble résulter du développement d'un remodelage cardiaque responsable d'une rigidité myocardique et d'une baisse de la contractilité.

Mots clés : Excès calorique / adiposité abdominale / fonction cardiaque / composition des phospholipides / réserve coronaire

\section{Introduction}

Obesity is associated with increased risk of cardiovascular diseases (Mathers and Loncar, 2006) and improved survival after myocardial infarction and stroke (Romero-Corral et al., 2006). It is known to favor development of insulin resistance and type-2 diabetes which finally leads to diabetic cardiomyopathy (Picard et al., 2013) characterized by hypertrophy and/or fibrosis (Habbout et al., 2013a; Habbout et al., 2013b), atrial fibrillation (Andrade et al., 2014) and decreased cardiac pump activity (Habbout et al., 2013a). Recently, the progression of cardiac function during the development of obesity has been clearly characterized in animal models of postnatal overfeeding. Post-natal overfeeding is achieved in mice and rats by drastically decreasing the number of pups in a litter. This allows the pups to receive a higher quantity of milk which is also of better quality. Post-natal overfeeding increases the body weight and abdominal adiposity during animals' lifespan. In vivo cardiac function has been extensively studied at different ages (Habbout et al., 2013b). In 3-month old rats, ejection fraction increases. Afterwards, it progressively decreases and after the age of 9 months it becomes lower than that of control animals. Furthermore, the effects of high-fat diet-induced abdominal obesity on cardiac function have also been studied ex vivo in different models of perfused hearts (Marciniak et al., 2014; Burgmaier et al., 2010). The results from those studies clearly show that ex vivo cardiac function is rapidly reduced soon after the beginning of the high-fat (HF) diet. The main reason for that decrease is a reduction of mitochondrial metabolic efficiency related to increased amount of uncoupling protein-3 (Cole et al., 2011). Discrepancies between the in vivo and ex vivo measurements at the onset of obesity necessitates further investigation before a definitive conclusion can be drawn.

Western-type diet characterized by excess lipid intake and the lack of physical activity are responsible for the obesity pandemic occurring in the industrials and emerging countries (Coehlo et al., 2011). This diet is also characterized by a high ingestion of saturated fatty acids (SFA) and a low intake of polyunsaturated fatty acids (PUFA), mainly n-3 PUFA. The changes in the ingested fats in terms of quantity and quality can have a strong influence on cardiac membrane lipid composition and function. For example, n-3 PUFA can be incorporated in cardiac phospholipids and have a noticeable cardio-protective effect (Demaison et al., 1994). However, the effects of SFA and monounsaturated fatty acids (MUFA) on cardiac membrane lipid composition and cardiac function are less known.

This study was aimed at determining whether high dietary energy intake provided by equilibrated and unbalanced diets in terms of lipid composition can modify the fatty acid profile of cardiac phospholipids and function of cardiac cells and at examining if the changes in membrane lipid composition can explain cellular activity modifications. High energy intake was achieved through two distinct ways. The first was achieved by feeding Wistar rats a HF diet rich in SFA and MUFA, which is known to increase energy intake. Second, in the Zucker diabetic Fatty (ZDF) rat fed an equilibrated diet. ZDF rats eat much more than their lean littermates (Zucker lean rats or ZL). The fatty acid composition of cardiac phospholipids was determined and several cardiovascular risk markers were evaluated in the heart itself (eicosapentaenoic acid (EPA) to arachidonic acid (AA) ratio as well as sum of EPA and docosahexaenoic acid (DHA) in membrane phospholipids) and in the blood (glycemia, insulinemia, proportion of glycated hemoglobin, triglyceridemia, total cholesterolemia). Finally, the function of different cardiac cells (endothelial and smooth muscle cells of the micro-vessels, cardiomyocytes) was measured and several experiments were conducted to elucidate the impact of changes in membrane lipid composition on cellular function.

\section{Materials and methods}

\subsection{Animals and diets}

All experiments followed the European Union recommendations concerning the care and use of laboratory animals for experimental and scientific purposes. All animal work was approved by the local board of ethics for animal experimentation (Cometh) and notified to the research animal facility of our laboratory (authorization $n^{\circ} 380723$ ).

In a first set of experiments, 60 male Wistar rats were housed 2 per cage in our animal facility at the age of 3 months. They were divided in two groups in order the mean of their body weight was similar and each group was then fed with a specific diet. The high fat diet-fed (HF) group was fed with a diet containing $31.5 \%$ of proteins, $54 \%$ of lipids (50\% of lard, $4 \%$ soya-bean oil w/w) and $7 \%$ of cellulose for a twelve week period. The control group was nourished with a commerciallyavailable diet (A04, Safe, Augy, France) which contained $16.1 \%$ of proteins, $3.1 \%$ of lipids and $60 \%$ cellulose. The energy from fat in the HF diet typically represents more than $50 \%$ of total calories as in an average Western diet. After analysis of the fatty acid composition of the diets, we found that the standard diet contained $24 \%$ of saturated fatty acids (SFAs), $23 \%$ of monounsaturated fatty acids (MUFAs), $48 \%$ of $n-6$ polyunsaturated fatty acids (PUFAs) and $4.5 \%$ of n-3 PUFAs while the HF diet contained $37 \%$ of SFAs, $46 \%$ of MUFAs, $15 \%$ of n-6 PUFAS and $1.2 \%$ of n-3 PUFAs. All groups were fed 
ad libitum with free access to water. It should be noted that the protein content of the HF diet was 2-fold higher because of the lower food intake (g/day) of these rats due to the high energy density. This allowed a similar daily protein intake in all groups.

In a second set of experiments, 10 Zucker Diabetic Fatty (ZDF) and 10 Zucker lean (ZL) rats were obtained from Charles River (L'Arbresle, France) at 7 weeks of age. They were housed 2 or 3 per cage in our animal facility and fed ad libitum with the commercially-available diet for 4 weeks.

On the day of the experiment, the rats were weighed and heparinized $(1500 \mathrm{IU} / \mathrm{kg})$ intraperitoneally $30 \mathrm{~min}$ before heart collection. Blood samples were collected for further biochemical analysis and their retroperitoneal and mesenteric adipose tissues were weighed for determination of abdominal fat mass.

\subsection{In vivo cardiac function study}

After deep anesthesia with sodium pentobarbital $(40 \mathrm{mg} / \mathrm{kg}$ ), the animal throat was dissected in order to isolate the right carotid artery. That artery was clamped at the proximal level in order to stop the blood flow arriving from the heart and a pressure gauge (Millar Instruments Inc., Houston, Texas) related to an amplifier (Gould) was introduced downstream through a small incision in the vessel. The clamp was removed and the gauge was progressively introduced in the aorta and then in the left ventricle cavity. The left ventricle pressure was then monitored after a 10-min period of stabilization. The heart rate, systolic, diastolic, developed pressures, $\mathrm{d} P / \mathrm{d} t$ max and $\mathrm{d} P / \mathrm{d} t$ min were determined from the recordings.

\subsection{Ex vivo cardiac function}

All rats underwent ex vivo Langendorff assessment of their cardiac function. For this reason, a rapid thoracotomy was performed and the heart was immediately collected in KrebsHeinselet solution maintained at $4{ }^{\circ} \mathrm{C}$. It was then rapidly (less than $1 \mathrm{~min}$ ) perfused according to the Langendorff mode with a Krebs-Heinselet buffer containing (in $\mathrm{mM}$ ) $\mathrm{NaCl} 119, \mathrm{MgSO}_{4}$ (1.2), $\mathrm{KCl} 4.8, \mathrm{NaHCO}_{3} 25, \mathrm{KH}_{2} \mathrm{PO}_{4} 1.2, \mathrm{CaCl}_{2} 1.2$ and glucose 11 plus hexanoate 0.5 as energy substrates. The buffer was maintained at $37{ }^{\circ} \mathrm{C}$ and continuously oxygenated with carbogen $\left(95 \% \mathrm{O}_{2} / 5 \% \mathrm{CO}_{2}\right)$. A latex balloon connected to a pressure probe was inserted into the left ventricle and filled until the diastolic pressure reached a value of $10 \mathrm{mmHg}$. This allowed the monitoring of heart rate, systolic, diastolic and left ventricle developed pressure (LVDP) throughout the perfusion protocol. A pressure gauge inserted into the perfusion circuit just upstream the aortic cannula allowed the evaluation of the coronary pressure. Except the ZDF and ZL rats which were allowed to beat at their spontaneous rhythm, the hearts were always electrically paced at a rate of 375 beats/min. When the hearts were electrically paced, cardiac mechanical work was considered to be represented by the LVDP. When they were not paced, it was estimated by the rate pressure product (heart rate $\times$ LVDP), since the heart rate was different in each perfused organ.
In a first set of experiments, the hearts were perfused at fixed pressure $(60 \mathrm{mmHg})$ and the mechanical function was determined after $30 \mathrm{~min}$. In that model of fixed pressure, the coronary flow slightly but gradually declined and this could impact the oxygen and substrate supply to the myocardium. In order to eliminate that negative influence, we decided to perfuse at fixed coronary flow in a second set of experiments. The coronary flow was determined at the 5 th $\min$ of perfusion in order the coronary pressure reached a value of $65 \mathrm{mmHg}$ and it was then maintained constant at this value for the 30 -min perfusion by means of a peristaltic pump. The temperature of the perfusion fluid was perfectly regulated in order to reach $37^{\circ} \mathrm{C}$ when the buffer arrived to the heart. This was allowed by pre-calibrating the temperature of the stock buffer solution as a function of the perfusion flow. Furthermore, the heart was immerged in a Krebs-Heinselet solution strictly maintained at $37{ }^{\circ} \mathrm{C}$. During the 30 min perfusion, the coronary pressure slightly increased, but the augmentation was minimal (never more than $5 \mathrm{mmHg}$ ). The mechanical function of the hearts was evaluated at the end of the 30 -min perfusion.

\subsection{Ex vivo coronary reactivity}

The coronary reactivity was evaluated in the ex vivo situation as previously described (Mourmoura et al., 2013). After 30 min of fixed flow perfusion, the coronary flow was maintained constant by means of a peristaltic pump and the coronary pressure was artificially increased for $20 \mathrm{~min}$ by infusion of a thromboxane A2 analog (U46619, $30 \mathrm{nM})$. U46619 infusion was maintained and acetylcholine or sodium nitroprusside was injected at various doses $(4,10,20,40,60,80$ and 100 or $100,200,400,600,800$ and 1000 pmoles) for determination of endothelial-dependent (EDV) or endothelial-independent (EIV) vasodilatations, respectively. The vasodilatation activity of endothelial cells (VAEC) was then calculated from the two curves. In order to simplify the understanding of the results, the EDV and VAEC was presented only when 60 pmoles of acetylcholine were injected. Similarly, EIV was presented only for the nitroprusside dose of 200 pmoles.

The EDV, EIV and VAEC were determined in basal conditions and after pre-treatment of the hearts with indomethacin $(2.5 \mu \mathrm{M})$, a cyclooxygenase inhibitor.

\subsection{Cardiac mitochondrial oxidative stress}

The ratio between the activities of aconitase and fumarase of the myocardium was calculated as an indicator of mitochondrial reactive oxygen species (ROS) impregnation. Mitochondrial aconitase is sensitive to inactivation by ROS whereas fumarase is not. Aconitase and fumarase activities were evaluated according to Gardner et al. (1994).

\subsection{Fatty acid composition of cardiac phospholipids}

It was determined as previously described (Demaison et al., 1994). The lipids were extracted according to Folch et al. (1957) and the phospholipids were separated from 
Table 1. Morphological and biochemical data.

\begin{tabular}{ccccc}
\hline & Control & HF & ZL & ZDF \\
\hline Energy intake (kcal/day/100 g) & $14.9 \pm 0.3$ & $16.4 \pm 0.2^{*}$ & $15.8 \pm 1.2$ & $29.6 \pm 4.0^{*}$ \\
Body mass (g) & $438 \pm 7$ & $472 \pm 19$ & $300 \pm 1$ & $364 \pm 2^{*}$ \\
Abdominal adiposity (g/100 g bw) & $2.7 \pm 0.3$ & $4.9 \pm 0.3^{*}$ & $1.3 \pm 0.1$ & $3.9 \pm 0.1^{*}$ \\
Heart weight (mg/100 g bw) & $186 \pm 6$ & $207 \pm 9$ & $171 \pm 6$ & $175 \pm 18$ \\
\hline Insulin $(\mathrm{IU} / \mathrm{l})$ & $1.3 \pm 0.1$ & $1.1 \pm 0.2$ & $1.3 \pm 0.1$ & $4.5 \pm 0.8^{*}$ \\
OGTT (AUC glucose) & $29077 \pm 1048$ & $32298 \pm 1167^{*}$ & - & - \\
HbA1c $(\%)$ & - & - & $4.1 \pm 0.1$ & $9.2 \pm 0.3^{*}$ \\
Glycemia (mM) & $5.1 \pm 0.3$ & $5.2 \pm 0.1$ & $6.0 \pm 0.4$ & $24.3 \pm 1.3^{*}$ \\
Triglyceridemia $(\mathrm{g} / \mathrm{l})$ & $1.0 \pm 0.1$ & $1.4 \pm 0.1 *$ & $0.9 \pm 0.1$ & $1.8 \pm 0.1^{*}$ \\
Total cholesterolemia $(\mathrm{g} / \mathrm{l})$ & $0.53 \pm 0.01$ & $0.88 \pm 0.06^{*}$ & $0.76 \pm 0.01$ & $1.37 \pm 0.05^{*}$ \\
\hline
\end{tabular}

The number of experiments was 10 per group. HF: high fat fed rats; ZL: Zucker lean rats; ZDF: Zucker Diabetic Fatty rats; bw: body weight; OGTT: oral glucose tolerance test; AUC: area under the curve expressed in arbitrary units; HbA1c: glycated hemoglobin; -: missing data; *: significantly different between HF and control rats or ZL and ZDF animals.

non-phosphorus lipids using a Sep-pack cartridge (Juaneda and Rocquelin, 1985). After transmethylation, the fatty acid methyl esters were separated and analyzed by gas chromatography.

\subsection{Other biochemical determinations}

Blood glucose concentrations were determined with a glucose analyzer (ACCU-CHECK Active, Softclix). Plasma insulin was evaluated using a radio-immunoassay kit (ICN Pharmaceuticals, Orangeburg, SC). Plasma triglycerides and cholesterol levels were estimated using kits from Biomérieux (Craponne, France) and Roche (BoulogneBillancourt, France). Glycated hemoglobin (HbA1c) levels were evaluated with the kit Bayer Healthcare's analyser A1cNow (Romans, France).

\subsection{Statistical analysis}

Results are presented as mean \pm S.E.M. Animal weight, heart dry weight, abdominal adiposity, glycemia, aconitaseto-fumarase ratio, fatty acid composition of cardiac phospholipids, data describing the cardiac mechanical and vascular function were contrasted across the two groups by one-way analysis of variance. Measures related to the action of indomethacin were treated with two-way analysis of variance to test the effects of the diet and that of indomethacin. When required, group means were contrasted with a Fisher's LSD test. A probability $(p)$ less than 0.05 was considered significant. Statistical analysis was performed using the NCSS 2007 software.

\section{Results}

\subsection{General data}

As shown in Table 1, the dietary energy intake was increased in the HF group $(+10 \%)$ compared to the control group. This slightly increased the body weight of the animals $(+8 \%)$, but the difference was not significant. However, the augmented energy intake strongly increased abdominal adiposity (sum of visceral and perirenal fats, $+81 \%$ ) and tended to augment the heart weight $(+11 \%)$. The parameters of glucose metabolism (glycemia and insulinemia) were not altered, but the higher circulating lipid content $(+40$ and $+66 \%$ for triglyceridemia and total cholesterolemia) contributed to increase glucose intolerance $(+11 \%)$.

The energy intake was also increased in ZDF animals $(+87 \%)$, but the pattern of morphological and biochemical parameters were different. Indeed, the body weight of the animals was significantly increased $(+21 \%)$ partly due to an augmented abdominal adiposity $(+200 \%)$. However, the heart weight was unaltered. Parameters of glucose metabolism were considerably increased with $+305 \%$ and $+246 \%$ augmentations of glycemia and insulinemia. The increase in those parameters clearly indicates the occurrence of glucose intolerance and insulin resistance, although these data were not determined in ZL and ZDF animals. Hyperglycemia was associated with accumulation of glycated hemoglobin $(+124 \%)$. ZDF animals also displayed increased plasma lipid contents with accumulation of triglycerides $(+100 \%)$ and total cholesterol $(+80 \%)$.

\subsection{Polyunsaturated fatty acid composition of cardiac phospholipids}

The proportions of polyunsaturated fatty acids of cardiac phospholipids were particularly altered in HF-fed and ZDF rats. Linoleic acid (C18:2 n-6) was decreased by $22 \%$ and arachidonic acid (C20:4 n-6) increased by $17 \%$ with the HF diet. This was even more pronounced in ZDF rats in which the reduction of linoleic acid reached $52 \%$ and increase in arachidonic acid $48 \%$. Those modifications were associated with unchanged proportions of eicosapentaenoic acids. As a consequence, the ratios between eicosapentaenoic and arachidonic acids were unaltered in the HF-diet fed rats and reduced by $41 \%$ in ZDF rats, clearly indicating the occurrence of an inflammatory situation in the last animals. Interestingly, the sum of eicosapentaenoic and docosahexaenoic acids (C22:6 n-3) was unaltered in HF-fed rats while it was increased in ZDF rats $(+94 \%)$, suggesting that those last animals were protected against sudden cardiac death.

When the adequate ratios between specific fatty acids were calculated, we came to the conclusion that high energy intake (HF diet and ZDF rats) stimulated elongases and inhibited $\Delta 5$-, $\Delta 6$ - and $\Delta 9$-desaturases. 
Table 2. Fatty acid composition of cardiac phospholipids.

\begin{tabular}{ccccc}
\hline & Control & HF & ZL & ZDF \\
\hline SFA & $38.8 \pm 0.2$ & $43.3 \pm 0.9^{*}$ & $35.9 \pm 0.8$ & $44.7 \pm 1.1^{*}$ \\
MUFA & $8.9 \pm 0.2$ & $7.1 \pm 0.1^{*}$ & $9.8 \pm 0.4$ & $6.2 \pm 0.2^{*}$ \\
PUFA & $52.4 \pm 0.1$ & $49.8 \pm 0.8^{*}$ & $54.2 \pm 0.6$ & $49.1 \pm 1.2^{*}$ \\
C18:2 n-6 & $26.5 \pm 1.3$ & $20.7 \pm 1.0^{*}$ & $32.9 \pm 1.8$ & $15.7 \pm 2.6^{*}$ \\
C20:4 n-6 & $17.0 \pm 0.3$ & $19.9 \pm 0.8^{*}$ & $16.0 \pm 0.8$ & $23.6 \pm 1.6^{*}$ \\
C22:4 n-6 & $0.2 \pm 0.1$ & $0.5 \pm 0.1^{*}$ & $0.4 \pm 0.1$ & $0.4 \pm 0.1$ \\
C22:5 n-6 & $0.2 \pm 0.1$ & $0.5 \pm 0.1^{*}$ & $0.3 \pm 0.1$ & $0.4 \pm 0.1$ \\
n-6 PUFA & $44.4 \pm 1.0$ & $41.9 \pm 0.3^{*}$ & $50.2 \pm 0.9$ & $41.2 \pm 1.3^{*}$ \\
C20:5 n-3 & $0.12 \pm 0.01$ & $0.10 \pm 0.02$ & $0.10 \pm 0.01$ & $0.08 \pm 0.06$ \\
C22:5 n-3 & $0.7 \pm 0.1$ & $1.8 \pm 0.1^{*}$ & $0.5 \pm 0.1$ & $1.3 \pm 0.1^{*}$ \\
C22:6 n-3 & $5.5 \pm 0.2$ & $5.4 \pm 0.4$ & $3.3 \pm 0.4$ & $6.5 \pm 0.5^{*}$ \\
n-3 PUFA & $6.4 \pm 0.2$ & $7.1 \pm 0.5$ & $4.0 \pm 0.5$ & $7.9 \pm 0.6^{*}$ \\
n-6/n-3 & $7.0 \pm 0.1$ & $5.9 \pm 0.4$ & $13.5 \pm 1.7$ & $5.4 \pm 0.5^{*}$ \\
EPA/AA & $6.8 \pm 0.8$ & $5.0 \pm 1.1$ & $6.1 \pm 0.5$ & $3.6 \pm 0.5^{*}$ \\
EPA+DHA & $5.7 \pm 0.2$ & $5.5 \pm 0.4$ & $3.4 \pm 0.4$ & $6.6 \pm 0.6^{*}$ \\
C18:0/C16:0 & $1.6 \pm 0.1$ & $2.5 \pm 0.1^{*}$ & $1.6 \pm 0.1$ & $1.9 \pm 0.1^{*}$ \\
C18:1n-7/C16:1n-7 & $10.9 \pm 1.6$ & $22.0 \pm 3.0^{*}$ & $10.4 \pm 0.5$ & $9.4 \pm 0.5$ \\
C20:2n-6/C18:2n-6 & $3.9 \pm 0.1$ & $10.3 \pm 0.7^{*}$ & $5.3 \pm 0.7$ & $12.8 \pm 3.2^{*}$ \\
C22:4n-6/C20:4n-6 & $1.4 \pm 0.1$ & $2.4 \pm 0.1^{*}$ & $2.4 \pm 0.1$ & $1.6 \pm 0.1^{*}$ \\
C22:5n-3/C20:5n-3 & $7.2 \pm 1.7$ & $25.4 \pm 5.6^{*}$ & $5.1 \pm 0.4$ & $15.6 \pm 1.6^{*}$ \\
C16:1n-7/C16:0 & $4.0 \pm 0.6$ & $1.2 \pm 0.1^{*}$ & $4.4 \pm 0.1$ & $2.7 \pm 0.1^{*}$ \\
C18:3n-6/C18:2n-6 & $7.8 \pm 0.5$ & $3.7 \pm 0.8^{*}$ & - & - \\
C20:4n-6/C20:3n-6 & $62.7 \pm 5.6$ & $33.4 \pm 2.7^{*}$ & $41.6 \pm 4.1$ & $25.2 \pm 4.7^{*}$ \\
\hline
\end{tabular}

The number of experiments was 10 per group. The EPA to AA ratio must be multiplied by $10^{-3}$. The C22:4 n-6/20:4 n-6 and C16:1 n-7/C16:0 ratios must be multiplied by $10^{-2}$. The C20:2 n-6/C18:2 n-6 and C18:3 n-6/C18:2 n-6 ratios must be multiplied by $10^{-3}$ and $10^{-4}$, respectively. HF: high fat fed rats; ZL: Zucker lean rats; ZDF: Zucker Diabetic Fatty rats; SFA: sum of saturated fatty acids; MUFA sum of monounsaturated fatty acids; PUFA: sum of polyunsaturated fatty acids; n-6/n-3: n-6/n-3 PUFA ratio; EPA: eicosapentaenoic acid or C20:5 n-3, AA: arachidonic acid or C20:4 n-6; DHA: docosahexaeboic acid or C22:6 n-3; -: missing data; *: significantly different between HF and control rats or ZL and ZDF animals.

Table 3. In vivo cardiac function in control and HF-fed rats.

\begin{tabular}{ccc}
\hline & Control & HF \\
\hline Heart rate (beats/min) & $367 \pm 7$ & $381 \pm 14$ \\
LVDP (mmHg) & $108 \pm 4$ & $135 \pm 10^{*}$ \\
$\mathrm{~d} P / \mathrm{d} t \max (\mathrm{mmHg} / \mathrm{s})$ & $3504 \pm 232$ & $4354 \pm 359^{*}$ \\
$\mathrm{~d} P / \mathrm{d} t \min (\mathrm{mmHg} / \mathrm{s})$ & $-3558 \pm 138$ & $-4189 \pm 338$ \\
Aconitase to fumarase ratio $(\%)$ & $47.9 \pm 1.4$ & $52.4 \pm 2.5$ \\
\hline
\end{tabular}

The number of experiments was 10 per group. HF: high fat; LVDP: left ventricle developed pressure; $\mathrm{d} P / \mathrm{d} t$ max describes the speed of contraction; $\mathrm{d} P / \mathrm{d} t$ min describes the speed of relaxation. *: significantly different.

\subsection{Ex vivo coronary reactivity}

EDV was determined in the hearts of control and HFfed rats in basal conditions or after pre-treatment with indomethacin, a cyclooxygenase inhibitor (Fig. 1). Upon basal conditions, the HF diet strongly increased EDV (+41\%). This was due to slight and non-significant increases in both ECVA and EIV ( +33 and $+20 \%$, respectively). Pre-treatment with indomethacin significantly reduced EDV in the HF group $(-47 \%)$ and deleted the differences between the two dietary groups. This was mainly explained by a reduction of EIV $(-41 \%)$.

\subsection{In vivo cardiac function}

Cardiac mechanical function was determined by introducing a pressure probe in the left ventricle. The heart rate was not altered by the HF diet, but the left ventricle developed pressure (LVDP) was significantly increased $(+25 \%)$. This was rather due to augmentation of the $\mathrm{dP} / \mathrm{dtmax}(+24 \%)$ than to improvement of the $\mathrm{dP} / \mathrm{dt} \min (+18 \%$ in absolute value). The HF diet-induced stimulation of cardiac mechanical activity was observed despite the aconitase to fumarase ratio which is inversely related to the mitochondrial oxidative stress was not affected by the diet.

\subsection{Ex vivo cardiac function}

As shown in Figure 2C, cardiac mechanical function was evaluated in ZDF rats and their lean littermates in a situation when the perfusion pressure was fixed. It was also monitored in HF-fed vs. control rats in two situations: when the hearts were perfused at fixed pressure (Fig. 2A) and when they were perfused at fixed coronary flow (Fig. 2E).

When the hearts were perfused at fixed pressure, the HF diet triggered a significant decrease in the mechanical work $(-34 \%)$. This was associated with a drop of the ratio between 


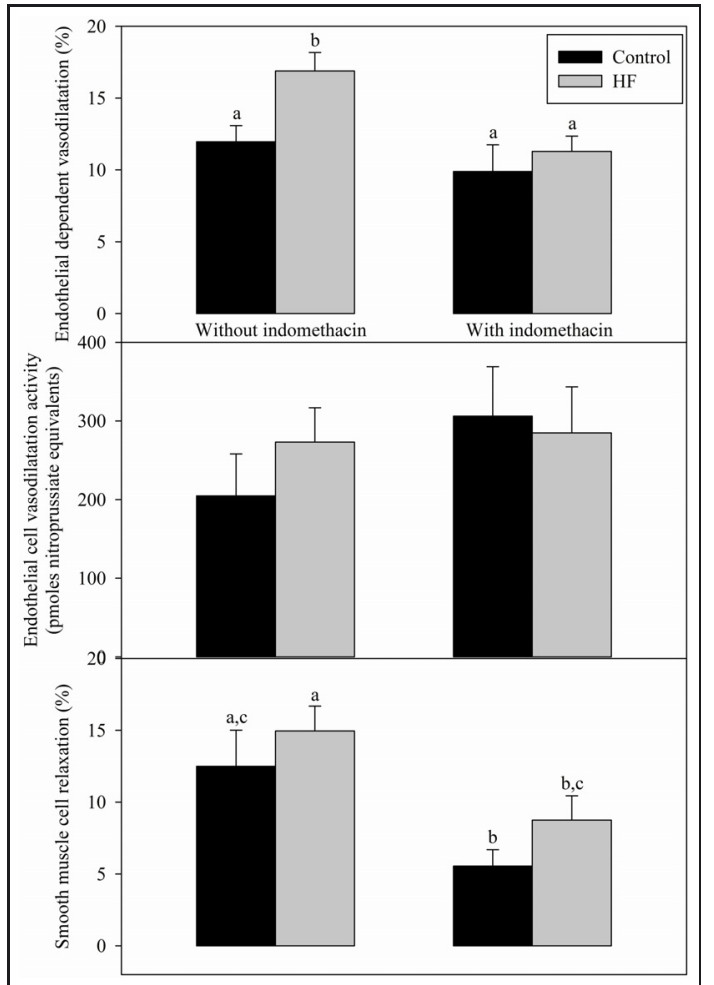

Fig. 1. Effects of the high fat (HF) diet on the endothelial-dependent vasodilatation (panel A) reflecting the integrative function of endothelial and smooth muscle cells, endothelial cell vasodilatation activity (panel B) and smooth muscle cell relaxation (panel C). The number of experiments was 10 per group. a, b, c: means with different letters on a same panel are signicantly different.

aconitase and fumarase activities $(-42 \%$, Fig. $2 B)$. Similar results were obtained in the non-paced ZDF rats compared to their lean littermates. Indeed, the mechanical work was reduced by $36 \%$ and the aconitase to fumarase ratio by $40 \%$. The situation was very different when the HF hearts were perfused at fixed coronary flow, since the mitochondrial oxidative stress was not increased and the cardiac mechanical work only tended to be decreased (-20\%, not significant).

\section{Discussion}

This study was aimed at determining whether high dietary energy intake through equilibrated and unbalanced diets in terms of lipid composition modify the fatty acid profile of cardiac phospholipids and function of various cardiac cells and at examining if the changes in membrane lipid composition can explain the modifications of cellular activity.

\subsection{Markers of cardiac health}

High energy intake both in the Wistar rat fed the HF diet and in the overfed ZDF animal triggered a decrease in linoleate and an increase in arachidonate proportions. This phenomenon was even more pronounced in the ZDF rat although that animal ate an equilibrated chow. This clearly indicates that the observed changes in n-6 PUFA composition were not due to the

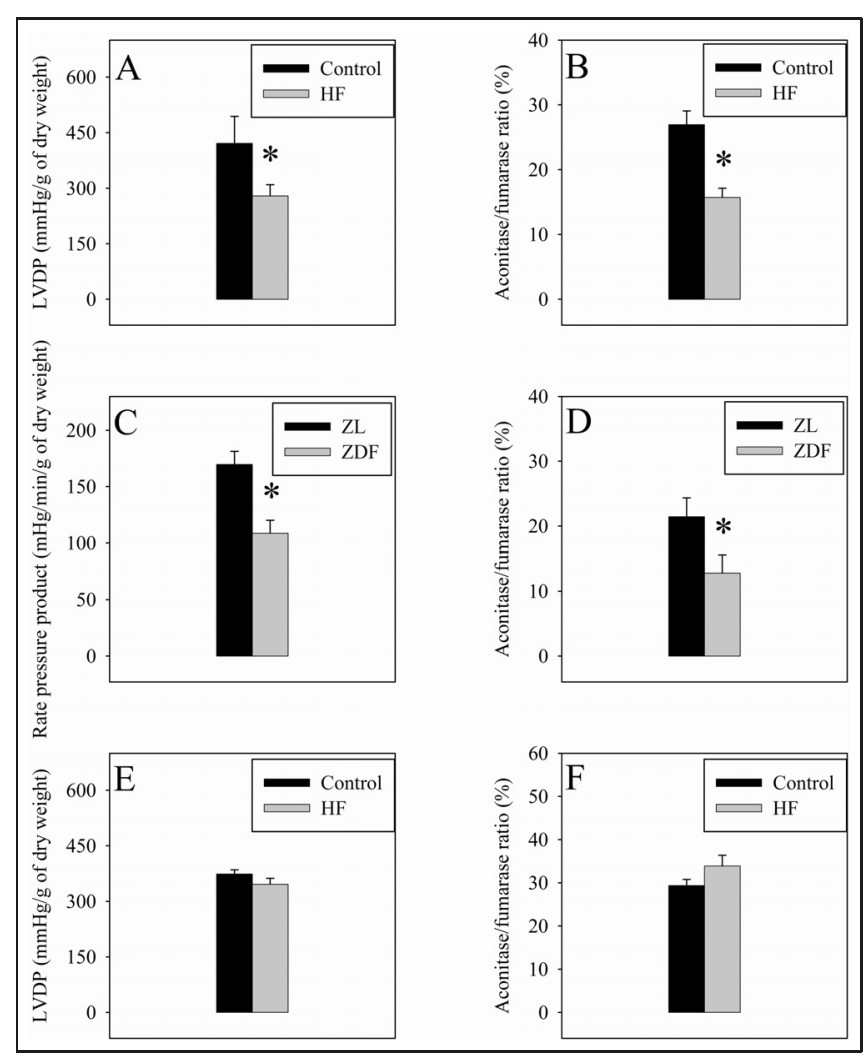

Fig. 2. Effects of high energy intake on the ex vivo cardiac mechanical function of hearts from Zucker Diabetic Fatty (ZDL) rats and their lean littermate (ZL, panel C) as well as high-fat (HF) diet fed rats and control animals perfused at fixed pressure (panel A) and at fixed coronary flow (panel E). The corresponding aconitase to fumarase ratio which is inversely proportional to the mitochondrial oxidative stress is located on the same line for each situation (panels B, D and F). The number of experiments was 10 per group. *: significantly different vs. control or ZL.

fatty acid content of the diet but rather to the amount of calories ingested. The results can be explained by a stimulation of elongases and inhibition of desaturases, which could contribute to the upholding of membrane integrity and fluidity. In contrast to the n-6 PUFA, the n-3 PUFA proportions of cardiac phospholipids were related to the dietary lipid composition. Indeed, the HF diet-fed rats eating a diet rich in SFA and MUFA and low in PUFA displayed unchanged proportions of total n-3 PUFA in cardiac phospholipids compared with the control animals. In contrast, the ZDF animals overfed the equilibrated chow had a higher percentage of n-3 PUFA in cardiac membranes (+98\%). Those differences affected the EPA to AA ratio of cardiac phospholipids leading to different inflammatory states (Rupp et al., 2004). In ZDF rats, this ratio was decreased by hyperphagy $(-41 \%)$, whereas it was unchanged in HF-fed rats. Interestingly, the sum of EPA and DHA whose decrease reflect the propensity to develop cardiac death (Rupp et al., 2004) was increased in the ZDF group $(+94 \%)$ whereas it was maintained in the HF group $(-4 \%)$. The alterations of EPA/AA ratios and EPA + DHA sums suggest that cardiac health was maintained in HF-fed rats (no inflammation and no risk of cardiac death). This clearly supports the new concept indicating that dietary saturated fatty 
acids are neutral concerning cardiovascular risk (Chowdhury et al., 2014). In ZDF animals, the lower EPA/AA ratio suggests development of an inflammatory process whereas the increased EPA + DHA sum indicates a lower propensity to display sudden cardiac death. These antagonistic results could favor atherosclerosis while being cardio-protective at the same time. This could explain the increased occurrence of infarction and augmented survival in obese individuals (Mathers and Loncar, 2006; Romero-Corral et al., 2006).

The ampler changes observed in ZDF rats compared to HF-fed rats are in agreement with the changes in plasma markers of cardiovascular health reported in the two types of overfed animals. Indeed, cholesterolemia and triglyceridemia were increased in both animal types, more in ZDF than in HF-fed rats, but hyperglycemia with increased HbAlc was noticed only in ZDF animals. ZDF rats could thus be more prone to cardiac diseases. However, the high amount of n-3 PUFA ingested by these animals could play a crucial role in maintaining a healthy myocardial environment.

\subsection{In vivo cardiac function and coronary reactivity}

Cardiac function in the in vivo situation was increased by the HF diet. This effect must be supported by an adaptation at the level of oxygen and substrate supplies arriving to the myocardium. We thus determined the coronary reactivity in HF-fed rats. The endothelium-dependent vasodilation was significantly enhanced in those animals, indicating that the capacity to augment the coronary flow was higher. This was due to a better function of endothelial and smooth muscle cells, but vasodilatation activity of endothelial cells was enhanced at a greater degree than the relaxation capacities of the smooth muscle cells.

In order to further understand the mechanism involved in that effect on the coronary micro-vessels, we investigated the effect of pre-treatment with indomethacin, a cyclooxygenase inhibitor. Interestingly, the increased endothelial-dependent vasodilatation observed in the HF group was totally suppressed with the pre-treatment. PUFA, mainly AA and EPA, are the substrates of cyclooxygenase. Their utilization leads to the formation of series- 2 and series- 3 prostanoids. Changing the PUFA composition of cardiac phospholipids is known to alter prostanoid production (Abayasekara and Wathes, 1999). Cyclooxygenase inhibition with indomethacin contributes to suppress prostanoid production, particularly PGI2 when AA proportion is high in cardiac phospholipids. PGI2 is a vasodilation agent which relaxes smooth muscle cells. Suppression of its production could thus prevent smooth muscle cells relaxation. In our study, we indeed observed that indomethacin reduced the relaxation of smooth muscle cells. The phenomenon contributed to erase the difference of endothelial-dependent vasodilatation induced by the HF diet. It is thus likely that the increased AA proportion in cardiac membrane of HF-fed rats facilitated PGI2 production, increased the relaxation of smooth muscle cells and ameliorated the endothelial-dependent vasodilatation. This effect supported the increased mechanical function observed in vivo, since higher supplies of oxygen and substrates are necessary to achieve this goal. However, it does not explain why the hearts of HF-fed rats displayed in vivo higher mechanical function. In order to know whether this increased cardiac function was due to the heart itself related to the increased AA proportion in membrane lipids or to environmental factors, we evaluated the cardiac function in the ex vivo situation.

\subsection{Ex vivo cardiac function}

Two Langendorff models were used to measure ex vivo cardiac function. They differed in their perfusion mode. The first model was a model of perfusion at fixed pressure, using the height of the perfusion column as a constant pressure regulator. This model is commonly used in cardiology. It allows a good monitoring of the cardiac function, but the coronary flow slightly and constantly decreases during the perfusion that may be related to perfusion fluid-related development of cellular edema. This can influence cardiac function. In our experiment, we noticed that ex vivo cardiac function of HF-fed rats was significantly lower than that of control animals, which strongly contrast with the in vivo situation. This was also true for ZDF rats compared to ZL animals. The measurement of the mitochondrial oxidative stress in that situation indicated a strong HF- and ZDF-induced increase in that parameter. This probably reduced Krebs cycle activity through inhibition of aconitase, decreased energy production and negatively affected inotropy (Mourmoura et al., 2013). We thus decided to utilize a second perfusion model in which the coronary flow was determined soon after the beginning of perfusion and maintained constant throughout it by means of a peristaltic pump. In that model, the perfusion temperature was strictly maintained at $37{ }^{\circ} \mathrm{C}$ during all the perfusion procedure. Under these perfusion conditions, the mitochondrial oxidative stress was similar in HF-fed and control rats resembling the in vivo situation. The ex vivo mechanical function was also decreased, but the magnitude of the reduction was lower than that measured with the fixed pressure perfusion and the difference was not significant.

Our results indicate that the increased cardiac function measured in HF-fed rats was not due to the heart itself, but rather to changes in environmental factors. Obesity is known to stimulate the adrenergic system (van Baak, 2001), which increases the delivery of catecholamines in the blood stream. These hormones exert a positive inotropic action on the heart which was facilitated by the AA-related increase in coronary reserve. The HF-induced increase in in vivo cardiac mechanical function was thus not due to the augmentation of AA proportion of cardiac phospholipids.

\subsection{Evolution of cardiac function during increased energy intake}

If stimulation of adrenergic system explains the increased inotropy at the early phase of obesity, it is not able to clarify the progressive cardiomyopathy occurring with prolonged obesity. In our experiment, cardiac dry weight normalized to animal growth (tibia length) tended to be increased by the HF diet $(+11 \%)$. This suggests that fibrosis and/or hypertrophy progressively occurred (Habbout et al., 2013b). HF diet is known to augment circulating angiotensin 2 which, once linked to its receptors, increase the expression of TGF $\beta-1$ 
(Rosenkranz, 2004). TGF $\beta$-1 induces the development of fibrosis and hypertrophy (Lin and Pan, 2008; Schlüter and Wenzel, 2008). Fibrosis and hypertrophy augment myocardial stiffness and reduce thus myocardial contractility. This can explain the cardiomyopathy occurring with long-term obesity.

\section{Conclusions}

High energy intake increased cardiac mechanical function in the in vivo situation. This was probably due to obesityrelated stimulation of adrenergic system which could depend on a change of neuronal and/or adrenal gland PUFA composition characterized by AA accumulation. The increased cardiac function was supported by an AA-related augmentation of the coronary reserve. This phenomenon could help the organism to sustain increased energy expenditure due to high body weight. With long-lasting obesity development, a cardiomyopathy with reduced mechanical function occurs. It seems to be due to development of fibrosis and/or hypertrophy which does not seem to be related to changes in cardiac phospholipid AA proportion. Our study showed that AA proportion in ZDF rats was strongly increased, but fibrosis and/or hypertrophy did not seem to develop in those animals. It should be interesting to know if angiotensin 2 production can be affected by phospholipid AA proportion of various organs containing an angiotensin-converting enzyme. More generally, it should be also interesting to know whether the decreased linoleate and increased arachidonate proportions are specific of the heart or can occur in other organs.

Acknowledgements. This work was financially supported by the French National Institute of Agronomical Research (INRA) and National Institute of Health and Medical Research.

\section{References}

Abayasekara DR, Wathes DC. 1999. Effects of altering dietary fatty acid composition on prostaglandin synthesis and fertility. Prostaglandins Leukot. Essent. Fatty Acids 61: 275-287.

Andrade J, Khairy P, Dobrev D, Nattel S. 2014. The clinical profile and pathophysiology of atrial fibrillation: relationships among clinical features, epidemiology, and mechanisms. Circ. Res. 114: 1453-1468.

Burgmaier M, Sen S, Philip F, et al. 2010. Metabolic adaptation follows contractile dysfunction in the heart of obese Zucker rats fed a high-fat "Western" diet. Obesity 18: 1895-1901.

Chowdhury R, Warnakula S, Kunutsor S, et al. 2014. Association of dietary, circulating, and supplement fatty acids with coronary risk: a systematic review and meta-analysis. Ann. Intern. Med. 160: $398-406$.

Coelho DF, Pereira-Lancha LO, Chaves DS, et al. 2011. Effect of high-fat diets on body composition, lipid metabolism and insulin sensitivity, and the role of exercise on these parameters. Braz. J. Med. Biol. Res. 44: 966-972.
Cole MA, Murray AJ, Cochlin LE, et al. 2011. A high fat diet increases mitochondrial fatty acid oxidation and uncoupling to decrease efficiency in rat heart. Basic Res. Cardiol. 106: 447-457.

Demaison L, Sergiel JP, Moreau D, Grynberg A. 1994. Influence of the phospholipid n-6/n-3 polyunsaturated fatty acid ratio on the mitochondrial oxidative metabolism before and after myocardial ischemia. Biochim. Biophys. Acta 1227: 53-59.

Folch J, Lees M, Sloane Stanley GH. 1957. A simple method for the isolation and purification of total lipides from animal tissues. $J$. Biol. Chem. 226: 497-509.

Gardner PR, Nguyen DD, White CW. 1994. Aconitase is a sensitive and critical target of oxygen poisoning in cultured mammalian cells and in rat lungs. Proc. Natl Acad. Sci. USA 91: $12248-12252$.

Habbout A, Li N, Rochette L, Vergely C. 2013a. Postnatal overfeeding in rodents by litter size reduction induces major shortand long-term pathophysiological consequences. J. Nutr. 143: 553-562.

Habbout A, Guenancia C, Lorin J, et al. 2013b. Postnatal overfeeding causes early shifts in gene expression in the heart and long-term alterations in cardiometabolic and oxidative parameters. PLoS One 8: e56981.

Juaneda P, Rocquelin G. 1985. Rapid and convenient separation of phospholipids and non phosphorus lipids from rat heart using silica cartridges. Lipids 20: 40-41.

Lin CS, Pan CH. 2008. Regulatory mechanisms of atrial fibrotic remodeling in atrial fibrillation. Cell. Mol. Life Sci. 65: 1489-1508.

Marciniak C, Marechal X, Montaigne D, Neviere R, Lancel S. 2014. Cardiac contractile function and mitochondrial respiration in diabetes-related mouse models. Cardiovasc. Diabetol. 13: 118.

Mathers CD, Loncar D. 2006. Projections of global mortality and burden of disease from 2002 to 2030. PLoS Med. 3: e442.

Mourmoura E, Vial G, Laillet B, et al. 2013. Preserved endotheliumdependent dilatation of the coronary microvasculature at the early phase of diabetes mellitus despite the increased oxidative stress and depressed cardiac mechanical function ex vivo. Cardiovasc. Diabetol. 12: 49.

Picard F, dos Santos P, Catargi B. 2013. Diabetes, obesity and heart complications. Rev. Prat. 63: 759-764.

Romero-Corral A, Montori VM, Somers VK, et al. 2006. Association of bodyweight with total mortality and with cardiovascular events in coronary artery disease: a systematic review of cohort studies. Lancet 368: 666-678.

Rosenkranz S. 2004. TGF-beta1 and angiotensin networking in cardiac remodeling. Cardiovasc. Res. 63: 423-432.

Rupp H, Wagner D, Rupp T, Schulte LM, Maisch B. 2004. Risk stratification by the "EPA+DHA level' and the "EPA/AA ratio" focus on anti-inflammatory and antiarrhythmogenic effects of long-chain omega-3 fatty acids. Herz 29: 673-685.

Schlüter KD, Wenzel S. 2008. Angiotensin II: a hormone involved in and contributing to pro-hypertrophic cardiac networks and target of anti-hypertrophic cross-talks. Pharmacol. Ther. 119: 311-325.

Van Baak MA. 2001. The peripheral sympathetic nervous system in human obesity. Obes. Rev. 2: 3-14.

\footnotetext{
Cite this article as: Evangelia Mourmoura, Valérie Chaté, Karine Couturier, Corinne Malpuech-Brugère, Kasra Azarnoush, Luc Demaison. Evolution from increased cardiac mechanical function towards cardiomyopathy in the obese rat due to unbalanced high fat and abundant equilibrated diets. OCL 2015, 22(4) D406.
} 